\title{
Enterococcus faecalis Enhances Candida albicans Mediated Tissue Destruction in a Strain-Dependent Manner
}

\author{
Akshaya Lakshmi Krishnamoorthy ${ }^{1,2}$ Alex A. Lemus ${ }^{3}$, Adline Princy Solomon ${ }^{2}$, Alex M. Valm \\ 3,4, Prasanna Neelakantan 1, * \\ 1 Faculty of Dentistry, The University of Hong Kong, Pok Fu Lam, Hong Kong \\ 2 Quorum Sensing Laboratory, Center of Research in Infectious Diseases, School of Chemical and \\ Biotechnology, SASTRA Deemed to be University, Thanjavur, India \\ 3 Department of Biological Sciences, University at Albany, State University of New York, Albany, NY USA \\ 4 The RNA Institute, University at Albany, State University of New York, Albany, NY USA \\ * Correspondence: prasanna@hku.hk; Tel.: +852-2859 0581
}

\begin{abstract}
Candida albicans as an opportunistic pathogen exploits the host immune system and causes a variety of life-threatening infections. The polymorphic nature of this fungus gives it tremendous advantage to breach mucosal barriers and cause a variety of oral and disseminated infections. Enterococcus faecalis, another opportunistic pathogen co-exists with $C$. albicans in several niches in the human body, including the oral cavity and gastrointestinal tract. However, interactions between E. faecalis and C. albicans on oral mucosal surfaces remain unknown. Here, for the first time, we comprehensively characterized the interactive profiles between laboratory and clinical isolates of $C$. albicans (SC5314 and BF1) and E. faecalis (OG1RF and 846) on an organotypic oral mucosal model. Our results demonstrated that the two species formed robust biofilms on the mucosal tissue surface with profound surface erosion and fungal invasion. Specifically, this effect was more pronounced in the laboratory isolates than in the clinical isolates. Notably, several genes of $C$. albicans involved in tissue adhesion, hyphal formation, fungal invasion, and biofilm formation were significantly upregulated in the presence of E. faecalis. This study highlights the strain-dependent cross-kingdom interactions between $E$. faecalis and C. albicans on oral mucosa, demonstrating the requisite to study more substrate-dependent polymicrobial interactions.
\end{abstract}

Keywords: biofilm; Candida albicans; E-cadherin; Enterococcus faecalis; FISH; oral mucosa.

\section{Introduction}

Candida albicans resides as a commensal organism in the human microbiota and exists in homeostasis with the microbial flora and epithelial tissues in healthy individuals [1]. However, it takes advantage of a weakened immune system and generates disturbances in such equilibrium, leading to a variety of recalcitrant and life-threatening infections. C. albicans is capable of forming tenacious biofilm communities on both biotic (mucosa) and abiotic surfaces (plastic surfaces of indwelling devices), each displaying dramatically different lifestyles, causing several oral infections including oropharyngeal candidiasis (OPC), dental caries and denture stomatitis [2].

C. albicans tightly adheres to oral epithelial cells, a phenomenon that is critical in establishing colonization. OPC (oral thrush) is a complication that occurs mostly due to the defects in host immune system, affecting more than $90 \%$ of the patients with progressive HIV/AIDS [3]. The ability of Candida albicans to switch from the yeast to hyphal form is the prime feature for invasion and pathogenesis. The strains that are unable to produce hyphae are considered non-invasive and avirulent, emphasizing the significant role of hyphae in mucosal infections [4]. Candidal invasion into the mucosal layer is promoted through several hyphal associated factors such as adhesion molecules (hyphal wall protein 1) and hydrolytic enzymes (phospholipases) [1]. C. albicans also interacts with 
the epithelia-associated proteins such as E-cadherin, which induces endocytosis and provides a mechanism of epithelial cell penetration [5].

The oral cavity serves as one of the first sites of entry for several pathogens. Therefore, bacterial and fungal species compete for colonization of the same niches (6). Several landmark discoveries of such synergistic and antagonistic behaviours of fungal-bacterial interactions have been reported in recent years. In extensive studies involving interactive profiles between Streptococci and C. albicans, it has been shown that $S$. oralis and C. albicans co-infection results in hypervirulent mucosal biofilms that significantly increase the frequency and size of oral thrush lesions [7]. On the other hand, interactions between Pseudomonas aeruginosa and C. albicans have been reported to be antagonistic, wherein the bacteria prevent hyphal formation by the fungus, in vitro [8]. Moreover, C. albicans responds effectively to the quorum sensing molecules produced by $P$. aeruginosa by secreting farnesol and thereby switching off its yeast to hyphal transition [9]. Therefore, comprehensive investigations of such interactive profiles between different bacteria and fungi is critical to the understanding of pathogenesis and the development of novel anti-microbial drugs [10].

Enterococcus faecalis, a Gram-positive bacterium is known to cause several infections including endocarditis, bacteraemia, abdominal abscesses, burn wound sepsis, meningitis, urinary tract infections and various nosocomial infections particularly in immunocompromised patients. Moreover, Enterococcus faecalis is an early colonizer and can form discrete microcolonies on the epithelial surface [11]. Furthermore, within the oral cavity, both E. faecalis and C. albicans are commonly implicated in the failure of root canal treatment. Hence, E. faecalis has a certain health care relevance and is involved in variety of polymicrobial infections [12].

Recently, a series of comprehensive and robust investigations demonstrated that $E$. faecalis OG1RF was antagonistic to Candida albicans SC5314 on abiotic surfaces. This was driven by the E. faecalis secreted bacteriocin, EntV, which inhibited C. albicans hyphal formation [13]. Furthermore, E. faecalis depletion in antibiotics treated mice attenuated C. albicans invasion [14], indicating that the former was required to invade mucosal tissues by the latter. These contrasting reports led us to question how different strains of these two species interact on mucosal tissue surfaces. Here, we hypothesised that C. albicans and E. faecalis act mutually and form robust mucosal biofilms, which cause tissue destruction. Using a variety of comprehensive investigations, we demonstrate that dual species biofilms resulted in tissue destruction and fungal invasion into mucosal compartments as same as the mono-species infections, in a strain-dependent manner.

\section{Materials and Methods}

\subsection{Chemicals, microbial strains, engineered tissue and culture conditions}

E. faecalis strains were grown overnight in Brain Heart Infusion Broth (BHI) under static conditions at $37^{\circ} \mathrm{C}$, while C. albicans strains were grown overnight in Yeast Potato Dextrose Broth (YPD) aerobically at $25^{\circ} \mathrm{C}$. The YPD medium consist of $5 \mathrm{~g} / \mathrm{L}$ of yeast extract, $10 \mathrm{~g} / \mathrm{L}$ of peptone, and $20 \mathrm{~g} / \mathrm{L}$ of dextrose. Reconstructed Human Oral Epithelium (RHOE) along with its maintenance media (MatTek Corporation, Ashland, Massachusetts, USA) were used in all the experiments. The assays were performed by growing the strains to mid- logarithmic phase and the OD595 was adjusted to achieve $\sim 1.5 \times 10^{8} \mathrm{CFU} / \mathrm{mL}$. The same culture conditions were maintained for all the experiments. All experiments were performed in triplicates as three independent experiments.

\subsection{Inoculation of mucosal tissues with C. albicans and E. faecalis}

Overnight cultures of E. faecalis and C. albicans were washed with Phosphate Buffered Saline (PBS) and resuspended in fresh media. Mucosal tissues were transferred to 6 well plates containing $0.9 \mathrm{ml}$ of pre-warmed MatTek assay medium per well. The microbial inoculum was prepared by inoculating $10^{7}$ cells of C. albicans and E. faecalis in PBS. $60 \mu \mathrm{l}$ of this inoculum was inoculated on the upper surface of the tissues and incubated for $18 \mathrm{~h}$. For certain assays, the tissues were incubated for 
$48 \mathrm{~h}$. The media was then collected, and the tissues were fixed with $10 \%$ formalin. Uninfected tissues were used as control in all experiments.

\subsection{Analysis of mucosal invasion and tissue destruction}

Formalin fixed tissues were dehydrated using a series of ethanol and xylene concentrations and then embedded in paraffin as described previously [15]. Tissue sections of $10 \mu \mathrm{m}$ thickness were obtained using a microtome and the sections were mounted on polysine slides (Rotary Microtome, Leica Rm2155, Wetzlar, Germany).

\subsubsection{Haematoxylin and eosin staining}

The slides mounted with the tissue sections were deparaffinized and stained with Haematoxylin and Eosin. The stained sections were visualised under the light microscope to observe the tissue architecture, biofilm formation and the invasion through mucosal layers [15].

\subsubsection{Quantification of tissue destruction using the Lactate Dehydrogenase (LDH) assay}

The amount of LDH released into the supernatant medium was monitored to indicate cell membrane integrity $[1,15]$. The supernatant was collected from the culture medium of the infected tissues and LDH activity was measured using a LDH cytotoxicity assay kit (Cayman Chemical Company, Ann Arbor, Michigan, USA) by measuring the OD490 and OD680 using a microplate reader (Spectramax M2 and M2e Multi-Mode Microplate Reader, Molecular Devices, San Jose, USA).

\subsubsection{Fluorescent in situ Hybridisation (FISH)}

Sections were labelled with fluorescence in situ hybridization probes as follows. Sections were labelled simultaneously with Caal probe for C. albicans, conjugated to Alexa fluor 488 (5'GCCAAGGCTTATACTCGCT-3') [16] and EUB 338 probe for E. faecalis conjugated to Alexa fluor 594 (5'-GCTGCCTCCCGTAGGAGT-3') [17]. Custom oligonucleotide probes were synthesized by Thermo Fisher Scientific (Waltham, MA, USA). FISH was carried out according to previously published protocols [18] except the hybridization time which was extended to $8 \mathrm{~h}$ based on our pilot studies for optimization. Hybridization solution [ $900 \mathrm{mM} \mathrm{NaCl}, 20 \mathrm{mM}$ Tris, $\mathrm{pH} 7.5,0.01 \%$ SDS 20\% (vol/vol) formamide] and both probes at a final concentration of $2 \mathrm{nM}$ ] were applied to the samples and incubated at $46^{\circ} \mathrm{C}$ for $8 \mathrm{~h}$ in a hybridization chamber. Slides were washed in wash buffer 1 [900 $\mathrm{mM} \mathrm{NaCl}, 20 \mathrm{mM}$ Tris, $\mathrm{pH} 7.5,0.01 \%$ SDS 20\% (vol/vol) formamide] at $48{ }^{\circ} \mathrm{C}$ for $15 \mathrm{~min}$ and then with wash buffer 2 [ $900 \mathrm{mM} \mathrm{NaCl}, 20 \mathrm{mM}$ Tris, $\mathrm{pH} 7.5,0.01 \%$ SDS] at $48{ }^{\circ} \mathrm{C}$ for $15 \mathrm{~min}$, and then dehydrated in an ethanol series, mounted in ProLong Gold antifade mountant with DAPI (Thermo Fisher Scientific) and covered with a \#1.5 coverslip. After curing overnight in the dark, the slides were imaged using a Zeiss LSM 710 confocal microscope with 20x 0.8 NA objective. Spectral images were acquired and then unmixed using the Zeiss Zen software and reference spectra were extracted from the images as described previously [19].

\subsection{Evaluation of tissue integrity using e-cadherin}

Immunofluorescent staining of E-cadherin was performed to evaluate the integrity of the mucosal layer challenged by the biofilms [20]. Deparaffinized sections were washed with PBS and antigen retrieval was achieved by treating the specimens with Saponin for 20 mins at $95{ }^{\circ} \mathrm{C}$, cooled slowly and washed with PBS for $10 \mathrm{~min}$. The sections were blocked using 10\% normal goat serum for $30 \mathrm{~min}$ at room temperature, and then incubated with mouse monoclonal primary antibody against E-cadherin at $37^{\circ} \mathrm{C}$ for $1.5 \mathrm{~h}$ (dilution ratio of 1:50). Subsequently, the unbound primary antibodies were removed by washing with PBS and the slides were incubated with the secondary antibody (Alexa Fluor 488 goat anti-mouse IgG) for 1 hour at room temperature under dark conditions. The samples were then mounted with a Fluoro-Gel II mounting medium containing DAPI. Slides were imaged using a Zeiss LSM 710 confocal microscope with $63 \times 1.4$ NA objective. Spectral images were acquired then unmixed using Zeiss Zen software and reference spectra extracted from the images as 
described above. Tissue autofluorescence spectral signatures were acquired from images of unstained tissue sections and subtracted from the images.

\subsection{Gene expression studies}

qRT-PCR based transcriptomic studies were performed to analyse the gene-expression changes in dual-species mucosal biofilms. The tissue sections were homogenized using PBS to remove the loosely adhered cells. Total RNA was extracted using the SV total RNA isolation system (Promega, Madison, Wisconsin, United States). The integrity and purity of total RNA was assessed by NanoDrop (Nanodrop 2000c, Thermo Fisher Scientific). cDNA was synthesized using the HighCapacity cDNA Reverse Transcription kit (Applied Biosystems, Foster City, CA, United States). Expression changes of the genes involved in biofilm formation, hyphal formation, virulence, and metabolism were analysed using qRT-PCR (Real-Time Pcr System, Stepone ${ }^{\mathrm{TM}}$ \& Steponeplus ${ }^{\mathrm{TM}}$, Applied Biosystems). Details of the primers has been tabulated (Tables S1 and S2). 18srRNA and 23srRNA were used as the reference genes for $C$. albicans and E. faecalis respectively. Fold changes in gene expression were calculated using the $2^{-\Delta \Delta C \mathrm{~T}}$ method.

\subsection{Statistical analysis}

All the assays were carried out in triplicates for three independent trials and the results were expressed as mean \pm SD. Statistical analysis of the data was performed by one-way ANOVA (Graph Pad Prism version 6.05). $\mathrm{p} \leq 0.05$ was considered statistically significant.

\section{Results and Discussion}

\subsection{Dual species biofilms of the laboratory reference strains are more invasive and destructive than the clinical isolates}

A schematic representation of the methods and results of this work has been shown (Fig. 1). It has been shown previously that $E$. faecalis attenuates $C$. albicans hyphae in vitro on abiotic substrates $[13,21]$. We asked if the same interactive profiles would manifest on biotic substrates such as mucosal tissues. Our results demonstrated that the surface erosion of tissues in the dual species biofilms of the reference strains (E. faecalis OG1RF and C. albicans SC5314) was similar to that observed with C. albicans alone, but significantly greater than with E. faecalis alone (Figs.2, 3). While large numbers and densely arranged hyphae were observed in the mono-species $C$. albicans and dual species challenged mucosa (reference strains), the dual species biofilms showed relatively less dense hyphal arrangement on the surface. We hypothesised that this may be due to the amplified tissue invasion by C. albicans. Indeed, this was validated by our FISH assay, which showed that $C$. albicans SC5314 penetrated deep into the mucosal tissue compartments in the dual species infections, compared to the mono-species (Fig. 3). E. faecalis alone is incapable of forming mucosal biofilms [12], which corroborated with our Haematoxylin and Eosin staining results. Thus, our results indicate that $E$. faecalis OG1RF acts mutually with C. albicans SC5314 and causes epithelial tissue invasion and destruction. Such mutualistic relationship has been reported between C. albicans and the commensal oral Streptococci. Moreover, similar to E. faecalis, S. oralis, displays a poor capacity to colonise mucosal surfaces and form biofilms alone [7].

We questioned if these results were true for human clinical isolates. Interestingly, Candida albicans BF-1, a strain obtained from an AIDS patient with nasopharyngeal carcinoma [22, 23], did not show dense hyphae in both mono-species and dual species models (Fig. 4). This was further confirmed by the results of our FISH assay, wherein the C. albicans BF1 strain was less invasive and very few cells could be observed on the mucosal surface. The E. faecalis 846 strain which only colonised in low cell density was neither invasive nor destructive as the OG1RF strain (Fig. 4). Taken together, the tissue destruction and fungal invasion were strain-dependent, wherein the clinical isolates were less invasive and less destructive compared to the reference strains [14]. 


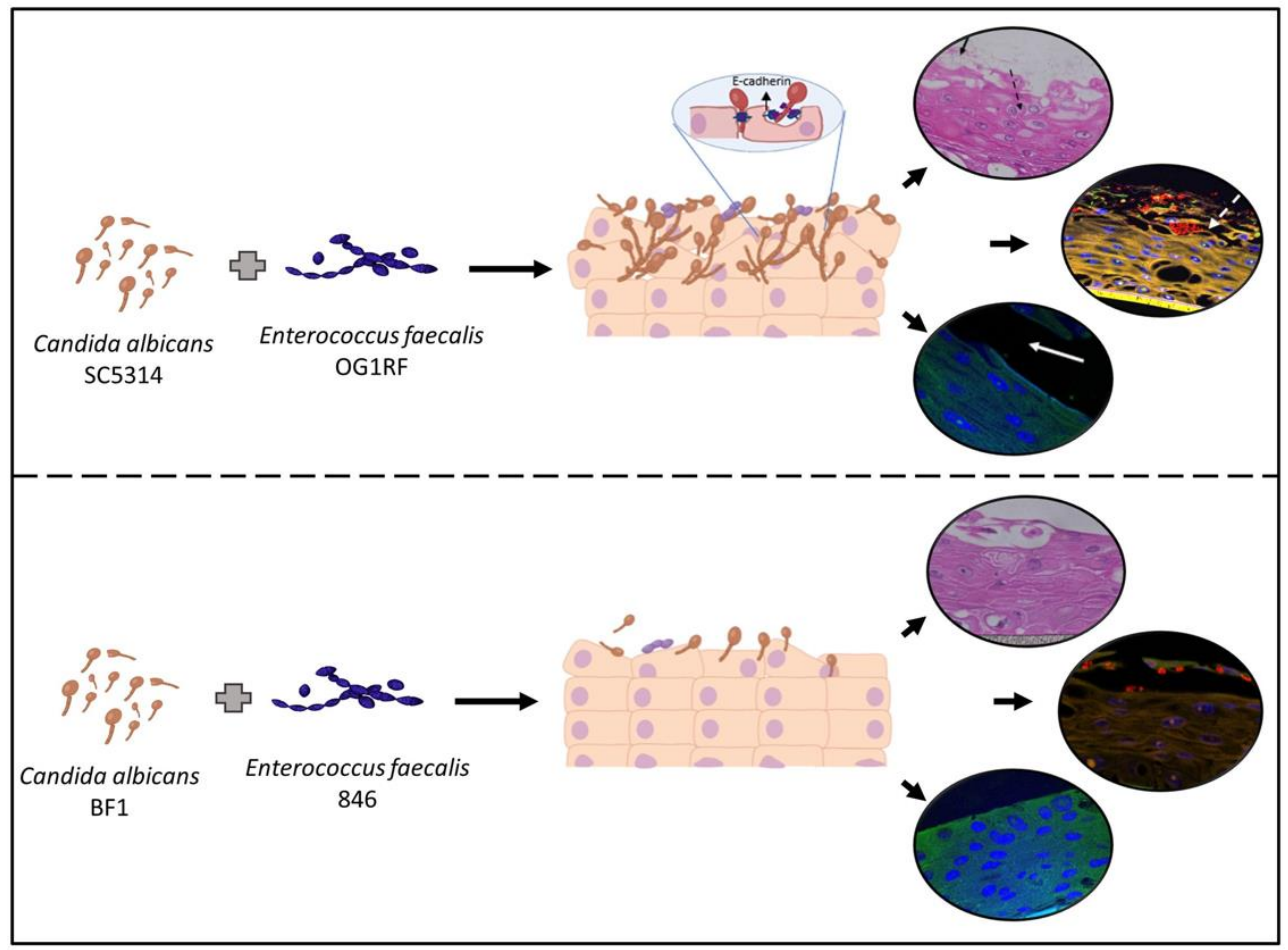

Figure 1. Schematic figure showing the hypothesis tested in the present study. The interactive profiles between the clinical isolates and laboratory isolates of C. albicans and E. faecalis were investigated using several qualitative and quantitative assays. The mucosal tissue invasion and erosion were significant with the laboratory strains, but not the clinical isolates.
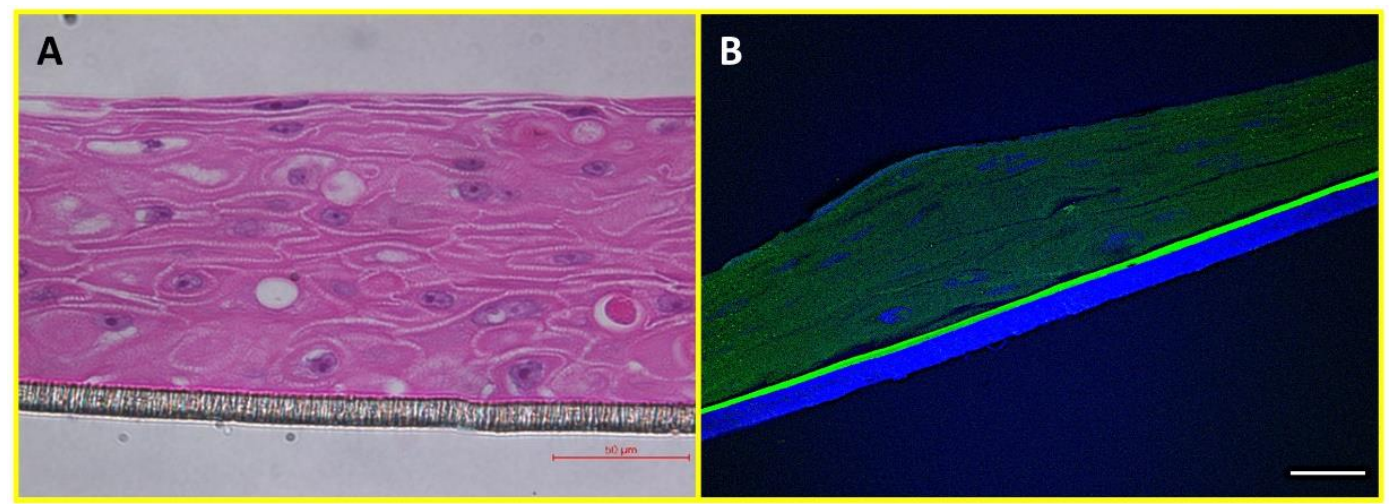

Figure 2. Cross-sectional images (x50) of the uninfected control tissues stained with Haematoxylin and Eosin and Immunofluorescence staining of E-cadherin are shown. In the E-cadherin stained tissue section, the scale bar is set to $25 \mu \mathrm{m}$ and the immunofluorescence staining was done using monoclonal antibody conjugated with Alexa Fluor 488 (green) and are counterstained with the nucleic acid stain DAPI (blue). 

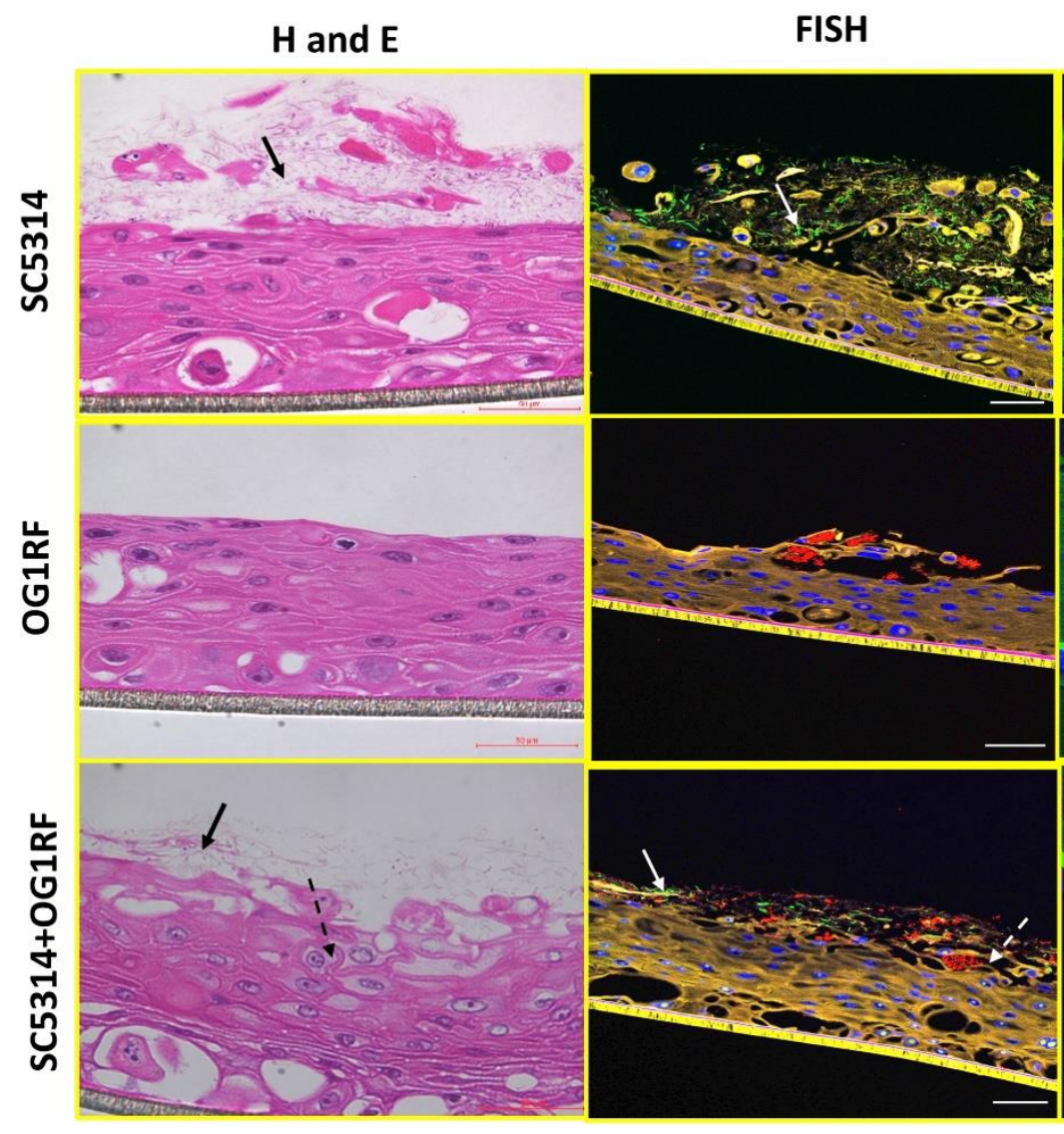

\section{E-cadherin}
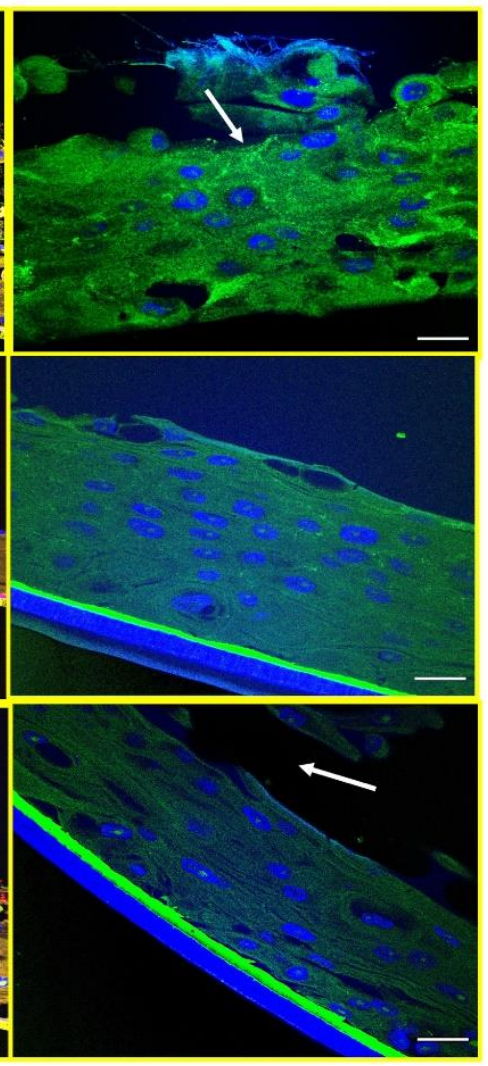

Figure 3. Cross-sectional images (x50) of the reference strains Candida albicans SC5314, Enterococcus faecalis OG1RF and Candida albicans SC5314 + Enterococcus faecalis OG1RF stained with Haematoxylin and Eosin, FISH and Immunofluorescence staining of E-cadherin are shown. Black arrows in the $\mathrm{H}$ and $\mathrm{E}$ stained sections indicates the hyphae on the biofilm and dotted arrow represents the loss in the tissue integrity. In the FISH-stained tissue sections $C$. albicans (green) was visualized after staining with Caal probe conjugated to Alexa fluor 488 and E. faecalis (red) was visualized after staining with a EUB 338 probe conjugated to Alexa fluor 594. White arrow in the FISH-stained tissue sections represent the hyphae on the biofilm, dotted arrow represents the loss in the tissue integrity and the scale bar is set to $50 \mu \mathrm{m}$. The immunofluorescence staining in the Ecadherin stained tissue sections was done using monoclonal antibody conjugated with Alexa Fluor 488 (green). White arrow in the E-cadherin (green) stained tissues indicates the degraded part of the tissue and the scale bar is set to $25 \mu \mathrm{m}$. In both FISH and E-cadherin-stained tissue sections, the mucosal cells were counterstained with the nucleic acid stain DAPI (blue). In multispectral FISH images, tissue autofluorescence is pseudocolored orange and tissue substrate materials are pseudocolored magenta and yellow. 

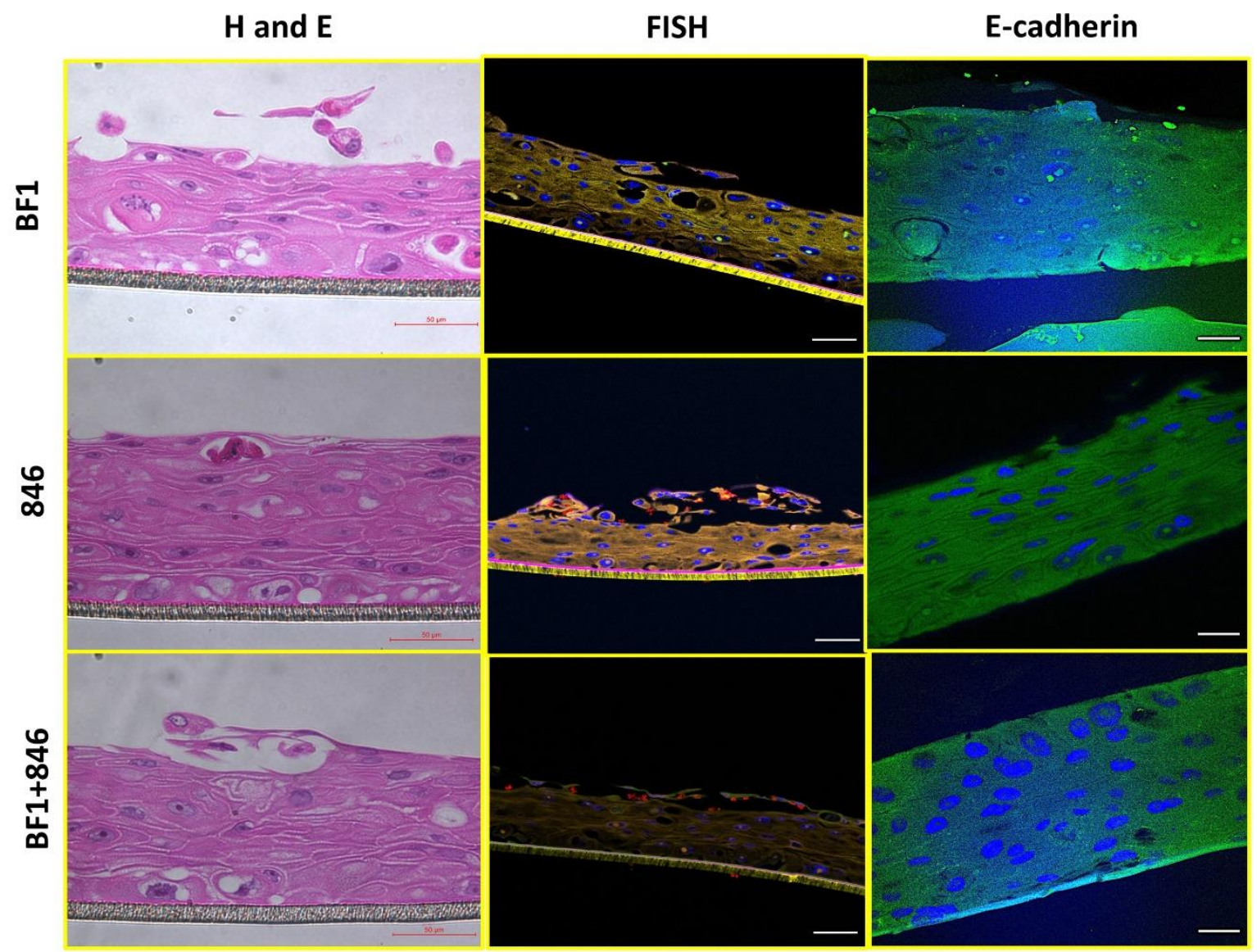

Figure 4. Cross-sectional images (x50) of the Clinical strains Candida albicans BF1, Enterococcus faecalis 846 and Candida albicans BF1 + Enterococcus faecalis 846 stained with Haematoxylin and Eosin, FISH and Immunofluorescence staining of E-cadherin are shown. In the FISH-stained tissue sections C. albicans (green) was visualized after staining with Caal probe conjugated to Alexa fluor 488 and $E$. faecalis (red) was visualized after staining with a EUB 338 probe conjugated to Alexa fluor 594. The scale bar is set to $50 \mu \mathrm{m}$. The immunofluorescence staining in the E-cadherin stained tissue sections was done using monoclonal antibody conjugated with Alexa Fluor 488 (green). The scale bar is set to $25 \mu \mathrm{m}$. In both FISH and E-cadherin-stained tissue sections, mucosal cells were counterstained with the nucleic acid stain DAPI (blue). In multispectral FISH images, tissue autofluorescence is pseudocolored orange and tissue substrate materials are pseudocolored magenta and yellow.

To quantify the effects of these biofilms on tissue destruction, the supernatant from the infected tissues ( $48 \mathrm{~h}$ ) was used for the LDH assay. After $48 \mathrm{~h}$ of incubation, there was complete tissue destruction, and thus, no tissues were available for histological or immunohistochemical assays (data not shown). The amount of LDH is used to describe the extent the tissue destruction. Dual species biofilms were characterised by significantly greater LDH quantity than E. faecalis, but not C. albicans mono-species biofilms (Fig. 5), indicating that E. faecalis did not attenuate C. albicans. Rather, these organisms mutually act and caused mucosal tissue destruction and fungal invasion similar to that of the mono-species model. 


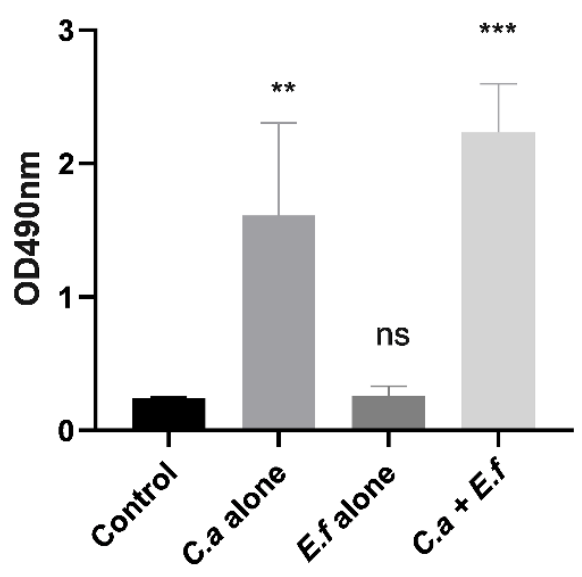

Figure 5. Lactate dehydrogenase $(\mathrm{LDH})$ released by the mucosal cells. Results represent the average OD490 of supernatant samples. ${ }^{* *}$ denotes $\mathrm{p} \leq 0.01$ and ${ }^{* * *}$ denotes $\mathrm{p} \leq 0.001$, and ns denotes $\mathrm{p}>0.05$. C.a C. albicans; E.f - E. faecalis

These results are in contrast with the findings of Graham et al., [13], who demonstrated that $E$. faecalis inhibited hyphal formation by $C$. albicans on abiotic substrates. Furthermore, the authors purified the peptide responsible for such effects and tested their potent antifungal effects in animal model. These contrasting results are noteworthy and scientifically important as they demonstrate the substrate-dependency and strain-dependency of microbial interactions. These findings go hand in hand with the mounting evidence that E. faecalis and C. albicans promote a mutually beneficial association with the host and that they coexist in human disease sites [32].

\subsection{Enterococcus faecalis - Candida albicans biofilms enhance E-cadherin degradation in mucosal compartments}

C. albicans adheres to and invades epithelial tissues by inducing endocytosis. To achieve this, the fungal hyphae express specific invasin-like molecules that bind to host cell receptors such as Ecadherin [25]. E-cadherin, a calcium dependent homophilic molecule, is important for cell-cell adhesion in epithelial tissues $[20,21]$. C. albicans invades into intercellular compartments in epithelial tissue by proteolytically degrading E-cadherin $[27,28]$.

To identify the potential mechanisms by which dual species biofilms caused increased tissue destruction, we asked if these biofilms degraded E-cadherin significantly more than mono-species biofilms. Interestingly, C. albicans SC5314 alone and in combination with E. faecalis OG1RF showed a marked degradation of the E-cadherin when compared with the uninfected control tissues and the $E$. faecalis infected tissues (Figs. 2,3). This assay indicated an exaggerated hyphal invasion into mucosal compartments in the dual species biofilm, supporting our hypothesis. The clinical isolate of E. faecalis did not demonstrate E-cadherin destruction. By contrast, the clinical isolate of $C$. albicans and dual species biofilms of the clinical isolates demonstrated E-cadherin degradation, although this was markedly less than the observations with the reference strains (Fig. 4). Further studies are required to quantify the tissue destruction with several clinical isolates.

\subsection{E. faecalis upregulates multiple C. albicans genes regulating adhesion, biofilm formation and virulence}

Our experiments on mucosal tissue invasion and degradation demonstrated that E. faecalis formed hypervirulent biofilms with $C$. albicans. Therefore, we tested the gene expression in the dual species mucosal biofilms, relative to the mono-species biofilms. Notably, E. faecalis dramatically upregulated (6-fold) ROB1, NDT80 and BRG1, the master regulatory genes involve in adhesion and biofilm formation in C. albicans [28], indicating that the former enhanced the ability of the latter to adhere to epithelial tissues and facilitated its biofilm growth (Fig. 6). Rather interestingly, gene 
expression of all the genes was downregulated at $48 \mathrm{~h}$, which suggest that these genes are involved only in the early stage of infection or only during biofilm formation. It is also possible that these genes were not upregulated due to the complete tissue destruction at $48 \mathrm{~h}$.

After the adherence phase, $C$. albicans invades epithelial tissue by inducing endocytosis, by expressing invasins such as $A L S 3$, which is regulated by the gene $A L S 3$. This gene was upregulated by $\sim 3$-fold in the dual species infections, suggesting that E. faecalis potentially enhanced the endocytosis of C. albicans [25]. Upregulation of the genes PLB1, PLB2 and SAP4 demonstrated that Candida albicans mucosal invasion is also mediated by the production of fungal aspartyl proteases and phospholipases [25,26]. Such effects have been reported for S. oralis - C. albicans biofilms [27]. These results corroborate with the findings from our E-cadherin assay as described previously. Both the E-cadherin and the FISH assays demonstrated hyphal invasion into mucosal compartments in the dual species biofilm [20]. Correspondingly, qRT-PCR analysis of the hyphal wall protein 1 (HWP1) showed an upregulation in dual-species biofilm model relative to C. albicans monospecies biofilms (Fig. 6). Taken together, our results indicate that E. faecalis supports the tissue degradation and invasion $C$. albicans by modulating its virulence genes.

Interestingly, all the tested genes of E. faecalis in the dual species biofilms were downregulated compared to the mono-species biofilm. E. faecalis has 3 quorum sensing systems - Fsr, Cytolysin and the inter-species communication system LuxS [29]. The two-component regulatory system consisting of the fsr locus in E. faecalis is critical for establishing its virulence. gelE, sprE, ef1097, and ef1097b are the four major genes that are directly dependent on the Fsr system, governing the production of gelatinase and serine protease [30]. Several post translational modifications of the genes gelE and sprE are required for activating EntV $[29,30]$. EntV, a bacteriocin produced by E. faecalis supresses hyphal morphogenesis of Candida albicans in vitro [13,21]. While this is true for abiotic substrates (e.g., dentures or catheters), our results demonstrate mutualistic interactions on mucosal tissue substrates. Expression of $g e l E, f s r B$ and $f s r C$ which are involved in the production of $E n t V$ were downregulated (5-fold).

The cylLL and cylLS genes are involved in the production of Cytolysin, which plays a major role in hemolysis, bacterial killing and other virulence traits of E. faecalis. Our results demonstrated that the presence of $C$. albicans resulted in $\sim 5$-fold downregulation of gelE, cylLS, luxS, esp, ace and efa. It has been shown that E. faecalis mediated killing of the nematode Caenorhabditis elegans was mitigated by E. faecalis strains that contained both cylLS and gelE [31], indicating a possible antagonistic effect of gelatinase and cytolysin. Therefore, it remains unclear if the downregulation of gelE and CylL in our results was attributed to the inherent antagonistic effect of C. albicans. The inllike gene of E. faecalis is responsible for the invasion of mammalian cells via E-cadherin. Our results showed a significant downregulation of this gene, explaining why E. faecalis alone was unable to destruct tissues or invade mucosal compartments. Indeed, based on the qualitative analyses in this study, it may not be possible to term this interaction as synergistic. However, the marked upregulation of many C. albicans genes responsible for tissue destruction and invasion in the presence of E. faecalis, indicates that these organisms act mutually on the mucosal surface and are not antagonistic to each other. 


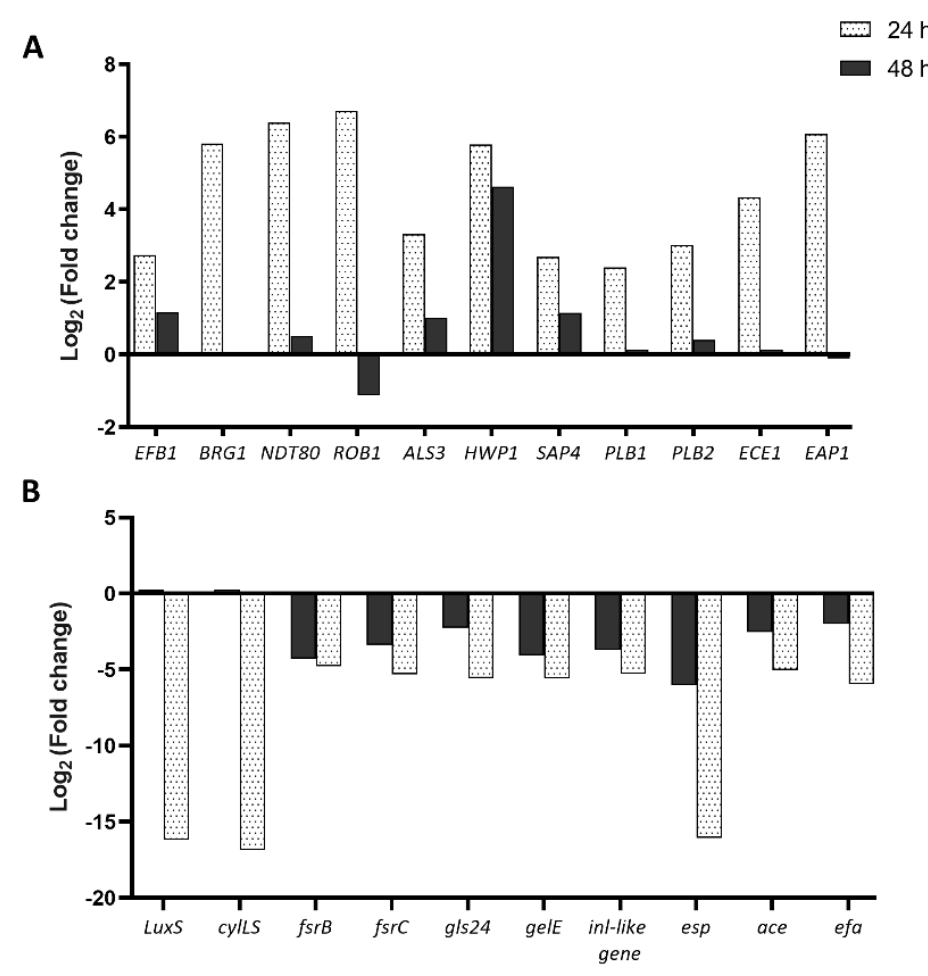

Figure 6. qRT-PCR analyses of different master regulatory genes present in the reference strain. (A) Logarithmic Relative change of the gene expression levels in the C. albicans alone and dual species mucosal model infected for 24 and 48 hours with 18srRNA as housekeeping gene (B) Logarithmic Relative change of the gene expression levels in E. faecalis alone and dual species mucosal model infected for 24 and 48 hours with 23srRNA as housekeeping genes

\section{Conclusions}

This study shines significant new light on the interactive profiles between E. faecalis and C. albicans on mucosal substrates. E. faecalis exaggerated the mucosal tissue invasion and destruction by C. albicans by upregulating several genes responsible for tissue adhesion, biofilm and hyphae formation and invasion. In this interaction, all the virulence genes of E. faecalis were downregulated, implying that the tissue destruction was not mediated directly by E. faecalis. These novel findings strongly suggest that multi-species biofilm communities should be investigated on a variety of substrates and environments.

Supplementary Materials: The following are available online at www.mdpi.com/xxx/s1, Table S1: Primer sequences of Candida albicans for qRT-PCR analysis, Table S2: Primer sequences of Enterococcus faecalis for qRTPCR analysis.

Author Contributions: Conceptualization, P.N, A.M.V and S.A.P.; methodology, A.L.K., A.A.L., P.N, A.M.V.; formal analysis, A.L.K, P.N, S.A.P., A.M.V.; investigation, A.L.K., A.A.L.; resources, P.N., A.M.V.; writingoriginal draft preparation, A.L.K., P.N.; writing-review and editing, P.N., S.A.P., A.M.V.; project administration, P.N. All authors have read and agreed to the published version of the manuscript.

Funding: This research received no external funding.

Acknowledgments: The work of Alex Valm is supported by a National Institutes of Health grant DE028042. The authors sincerely thank Becky P. K. Cheung, Geoffrey P.K Ng and Chui Y. Yip of the Central Research Laboratories, Faculty of Dentistry, The University of Hong Kong for the technical expertise and assistance in this work. The authors are indebted to Professor Anna Dongari-Bagtzoglou, University of Connecticut for the insightful discussions and the motivations to embark on this important and challenging project.

Conflicts of Interest: The authors declare no conflicts of interest. 


\section{References}

1. Dalle, F.; Wächtler, B.; L'Ollivier, C.; Holland, G.; Bannert, N.; Wilson, D.; et al. Cellular interactions of Candida albicans with human oral epithelial cells and enterocytes. Cell Microbiol. 2010, 12, 248-271. doi:10.1111/j.1462-5822.2009.01394.x.

2. Nett, J.E.; Marchillo, K.; Spiegel, C.A.; Andes, D.R. Development and validation of an in vivo Candida albicans biofilm denture model. Infect Immun. 2010, 78, 3650-3659, doi:10.1128/IAI.00480-10.

3. Vazquez, J.A. Options for the management of mucosal candidiasis in patients with AIDS and HIV infection. Pharmacotherapy. 1999, 19, 76-87, doi:10.1592/phco.19.1.76.30509.

4. Hasan, F.; Xess, I.; Wang, X.; Jain, N.; Fries, B.C.; Fries Biofilm formation in clinical Candida isolates and its association with virulence, Microbes Infect. 2009, 11(8-9): 753-761. doi:10.1016/j.micinf.2009.04.018

5. Naglik, J.R.; Richardson, J.P.; Moyes, D.L. Candida albicans Pathogenicity and Epithelial Immunity. PLoS Pathog. 2014, 10, 8-11. doi:10.1371/journal.ppat.1004257

6. Dongari-Bagtzoglou, A.; Kashleva, H.; Dwivedi, P.; Diaz, P.; Vasilakos, J. Characterization of Mucosal Candida albicans Biofilms. PLoS One. 2009, 4, e7967. doi:10.1371/journal.pone.0007967.

7. Xu, H.; Sobue, T.; Thompson, A.; Xie, Z.; Poon, K.; Ricker, A.; et al. Streptococcal co-infection augments Candida pathogenicity by amplifying the mucosal inflammatory response. Cell Microbiol. 2014, 16, 214231. doi:10.1111/cmi.12216.

8. Diaz, P.I.; Xie, Z.; Sobue, T.; Thompson, A.; Biyikoglu, B,; Ricker, A,; et al. Synergistic Interaction between Candida albicans and Commensal Oral Streptococci in a Novel In Vitro Mucosal Model. Infect Immun. 2012, 80, 620-632. doi:10.1128/IAI.05896-11.

9. Bandara, H.M.; Yau. J.Y.; Watt, R.M.; Jin, L.J.; Samaranayake, L.P. Pseudomonas aeruginosa inhibits in-vitro Candida biofilm development. BMC Microbiol. 2010, 10, 125. doi:10.1186/1471-2180-10-125.

10. Hogan, D.A.; Vik, Å., Kolter, R. A Pseudomonas aeruginosa quorum-sensing molecule influences Candida albicans morphology. Mol Microbiol. 2004, 54, 1212-1223. doi:10.1111/j.1365-2958.2004.04349.

11. Peleg, A.Y.; Hogan, D.A.; Mylonakis, E. Medically important bacterialg-fungal interactions. Nat Rev Microbiol. 2010, 8, 340-349. doi:10.1038/nrmicro2313.

12. Barnes, A.M.T.; Dale, J.L.; Chen, Y.; Manias, D.A.; Greenwood-Quaintance, K.E.; Karau, M.K. et al. Enterococcus faecalis readily colonizes the entire gastrointestinal tract and forms biofilms in a germfree mouse model. Virulence. 2017, 8, 282-296 doi: 10.1080/21505594.2016.1208890.

13. Graham, C.E.; Cruz, M.R.; Garsin, D.A.; Lorenz, M.C. Enterococcus faecalis bacteriocin EntV inhibits hyphal morphogenesis, biofilm formation, and virulence of Candida albicans. Proc Natl Acad Sci U S A. 2017, 114, 4507-4512 doi:10.1073/pnas.1620432114

14. Bertolini, M.; Ranjan, A.; Thompson, A.; Diaz, P.I.; Sobue, T.; Maas, K. et al. Candida albicans induces mucosal bacterial dysbiosis that promotes invasive infection. PLoS Pathog. 2019, 15, 1-30. doi:10.1371/journal.ppat.1007717.

15. Bertolini, M.M.; Xu, H.; Sobue, T.; Nobile, C.J.; Del Bel Cury, A.A.; Dongari-Bagtzoglou, A. Candidastreptococcal mucosal biofilms display distinct structural and virulence characteristics depending on growth conditions and hyphal morphotypes. Mol Oral Microbiol. 2015, 30, 307-322. doi:10.1111/omi.12095.

16. Kempf, V.A.J.; Trebesius, K.; Autenrieth, I.B.; Fluorescent in situ hybridization allows rapid identification of microorganisms in blood cultures. J Clin Microbiol. 2000, 38, 830-838. doi:10.1128/jcm.38.2.830-838.2000. 
17. Wang, Z.; Qi, Y.; Wang, J.; Pei, Y. Combination of 16S rRNA-targeted oligonucleotide probes with flow cytometry for analyzing mixed microbial populations. World J Microbiol Biotechnol. 2012, 28, 1919-1925. doi:10.1146/annurev.ecolsys.29.1.59.

18. Pernthaler, J.; Glöckner, F.O.; Schönhuber, W.; Amann, R. Fluorescence in situ hybridization (FISH) with rRNA-targeted oligonucleotide probes. Methods Microbiol. 2001, 30, 1-31. doi:10.1016/s05809517(01)30046-6.

19. Valm, A.M.; Mark Welch, J.L.; Rieken, C.W.; Hasegawa, Y.; Sogin, M.L. Oldenbourg R, et al. Systemslevel analysis of microbial community organization through combinatorial labeling and spectral imaging. Proc Natl Acad Sci U S A. 2011, 108, 4152-4157. doi:10.1073/pnas.1101134108.

20. Villar, C.C.; Kashleva, H.; Nobile, C.J.; Mitchell, A.P.; Dongari-Bagtzoglou A. Mucosal tissue invasion by Candida albicans is associated with E-cadherin degradation, mediated by transcription factor Rim101p and protease Sap5p. Infect Immun. 2007, 75, 2126-2135. doi:10.1128/IAI.00054-07.

21. Cruz, M.R.; Graham, C.E.; Gagliano, B.C.; Lorenz, M.C.; Garsin, D.A. Enterococcus faecalis inhibits hyphal morphogenesis and virulence of Candida albicans. Infect Immun. 2013, 81, 189-200. doi:10.1128/IAI.00914-12.

22. Seneviratne, C.J.; Wang, Y.; Jin, L.; Abiko, Y.; Samaranayake, L.P. Candida albicans biofilm formation is associated with increased anti-oxidative capacities. Proteomics. 2008, 8, 2936-2947. doi:10.1002/pmic.200701097.

23. Samaranayake, Y.H.; Samaranayake, L.P.; Pow, E.H.N.; Beena, V.T.; Yeung, K.W.S. Antifungal effects of lysozyme and lactoferrin against genetically similar, sequential candida albicans isolates from a human immunodeficiency virus-infected southern chinese cohort. J Clin Microbiol. 2001, 39, 3296-3302. doi:10.1128/JCM.39.9.3296-3302.2001.

24. Morse, D.J.; Wilson, M.J.; Wei, X.; Lewis, M.A.O.; Bradshaw, D.J.; Murdoch, C. et al. Denture-associated biofilm infection in three-dimensional oral mucosal tissue models. J Med Microbiol. 2018, 67, 364-375. doi:10.1099/jmm.0.000677.

25. Yang, W.; Yan, L.; Wu, C.; Zhao, X.; Tang, J. Fungal invasion of epithelial cells. Microbiol Res. 2014, 169, 803-810. doi:10.1016/j.micres.2014.02.013.

26. Liu. Y.; Filler, S.G. Candida albicans Als3, a multifunctional adhesin and invasin. Eukaryot Cell. 2011, 10, 168-173. doi:10.1128/EC.00279-10.

27. Xu, H.; Sobue, T.; Bertolini, M.; Thompson, A.; Dongari-Bagtzoglou, A. Streptococcus oralis and Candida albicans Synergistically Activate $\mu$-Calpain to Degrade E-cadherin from Oral Epithelial Junctions. J Infect Dis. 2016, 214, 925-934. doi:10.1093/infdis/jiw201.

28. Mayer, F.L.; Wilson, D.; Hube, B. Candida albicans pathogenicity mechanisms. Virulence. 2013, 4, 119128. doi:10.4161/viru.22913.

29. Ali, L.; Goraya, M.U.; Arafat, Y.; Ajmal, M.; Chen, J.L.; Yu, D. Molecular mechanism of quorum-sensing in Enterococcus faecalis: Its role in virulence and therapeutic approaches. Int J Mol Sci. 2017, 18, 960. doi:10.3390/ijms18050960.

30. Teixeira, N.; Varahan, S.; Gorman, M.J.; Palmer, K.L.; Zaidman-Remy, A.; Yokohata, R. et al. Drosophila Host Model Reveals New Enterococcus faecalis Quorum-Sensing Associated Virulence Factors. PLoS One. 2013. doi:10.1371/journal.pone.0064740.

31. La Rosa, S.L.; Solheim, M.; Diep, D.B.; Nes, I.F.; Brede, D.A. Bioluminescence based biosensors for quantitative detection of enterococcal peptide - Pheromone activity reveal inter-strain telesensing in vivo during polymicrobial systemic infection. Sci Rep. 2015, 5, 8339. doi:10.1038/srep08339. 
32. Garsin, D.A.; Lorenz, M.C. Candida albicans and Enterococcus faecalis in the gut: Synergy in commensalism?. Gut Microbes. 2013, 4, 409-415. doi:10.4161/gmic.26040 